Published in final edited form as:

AIDS Behav. 2014 February ; 18(0 2): 162-170. doi:10.1007/s10461-013-0474-4.

\title{
Sexual Health Knowledge and Health Practices of Female Sex Workers in Liuzhou, China, Differ by Size of Venue
}

\author{
Zhang Youchun, \\ School of Sociology and Population Studies, Renmin University of China, 58 Zhongguancun \\ dajie, Haidian, Beijing 100872, China
}

Jane D. Brown,

School of Journalism and Mass Communication, University of North Carolina at Chapel Hill, Chapel Hill, NC, USA

Kathryn E. Muessig,

School of Medicine, University of North Carolina at Chapel Hill, Chapel Hill, NC, USA

Feng Xianxiang, and

Liuzhou Center for Disease Control, Liuzhou, China

He Wenzhen

Department of Sociology, Tsinghua University, Beijing, China

Zhang Youchun: youchun_zhang@126.com

\section{Abstract}

We conducted qualitative interviews with 48 female sex workers (FSW) recruited from entertainment venues in Liuzhou, China. Analyses found that HIV knowledge and sexual health seeking strategies differed by size of venue: (1) Women in smaller venues said they douched before/after sex and used condoms with all but their regular partners and clients. Most found the brochures distributed by Chinese CDC workers "irrelevant" or "boring" and relied on friends for health advice. (2) FSW in middle and large venues were less concerned about prevention, claiming their clients were "healthy." They relied more on the Internet for health information and were less concerned about the cost of seeing a doctor. (3) Pregnancies and abortions were frequent, especially among the younger women in large venues. This research documents the need to develop tailored HIV-related messages and prevention strategies with the help of FSW to address differences among FSW working in venues of different sizes.

\section{Keywords}

Female sex workers; Health knowledge; Health practices; Venues; China

\section{Introduction}

A complex web of sexual health practices and industry-level factors place female sex workers (FSW) in China at increased risk for acquiring HIV/AIDS and other sexually transmitted diseases [1-4]. Although illegal, stigmatized and constantly under attack from police, prostitution remains a full-time job for many Chinese women. Interventions to improve sexual health practices among FSW in China tend to assume that women engage in

(C) Springer Science+Business Media New York 2013

Correspondence to: Zhang Youchun, youchun_zhang@126.com. 
high-risk behaviors because of low knowledge about correct condom use and HIV/STI transmission, and thus focus on education and condom-use skills training [1]. Previous research has shown, however, that while some FSW may continue to rely on ineffective prevention methods such as washing with salt water or disinfectants, douching, and selfmedicating with antibiotics [5], others are better informed about reproductive health and more skilled in condom use than women outside the sex trade [6]. Yet knowledge does not necessarily translate into safer sexual behaviors even among well-informed FSW [7, 8]. Instead, a variety of factors influence FSWs' choice or ability to practice safer sexual behaviors, including — but not limited to-economic hardship, concerns about fertility, client refusal/coercion, relationship status, and working conditions [8-13]. The relative importance of each of these factors for HIV/STI acquisition may also vary as they operate through the unique characteristics of different settings within the sex work industry $[2,5,7,13,14]$.

The Chinese Ministry of Health considers FSW a priority population for HIV/STI disease surveillance and prevention activities [15]. However, according to previous ethnographic research $[13,16]$, at least seven categories of Chinese sex workers exist, differing by workplace, types of services offered, income levels, and demographic characteristics. Recent epidemiologic surveillance data suggests that these differences may play a role in HIV/STI risk among FSW [4]. For example, in a study of 816 FSW, women recruited from streets and rented houses had a syphilis prevalence of $40.7 \%$ compared to $11.1 \%$ among women recruited from hair salons, saunas and massage shops [2]. Although national estimates of HIV prevalence among FSW remain relatively low $(<1 \%)$ [14], study samples have ranged as high as $10.3 \%$ among all FSW, and up to $49 \%$ among FSW who also use injection drugs [17]. This wide variation in prevalence across sex worker samples likely reflects these economic, social and structural differences in risk environments as well as geographic variation in the HIV epidemic in China and sampling strategies used across studies.

While rigorous evaluations of HIV-prevention interventions among FSW in China are limited, some have shown positive effects including improved HIV-related knowledge, increased condom use, and/or decreased STI infections [1, 18]. In other programs, however, FSW did not participate in organized prevention activities or utilize provided health services/information due to suspicion of government programs [5] and inconvenient, or inappropriate services [19]. In light of these findings and the broad variability across FSW populations described above, future interventions should be more multi-faceted and take into account environmental and structural factors that keep different groups of FSW from using services and engaging in prevention practices [1]. Accordingly, the current study was designed to identify appropriate elements to include in sexual health interventions for FSW in different types of work environments, and assess through which channels these interventions might be most effectively delivered. Our study specifically built on earlier reports that structural factors, including workplace organization and relationships with clients, affect the extent to which FSW in China can and do engage in healthy sexual practices $[7,13]$.

\section{Methods}

\section{Study Setting}

Among FSW in Liuzhou, HIV prevalence estimates range between 2.3 and $4.2 \%$ [20, 21], with substantially higher syphilis prevalence at $11 \%$ [20]. In 2005, the Women's Health Center (WHC) of the Liuzhou CDC was established to provide health education and intervention for FSW. According to the WHC, there were about 500 entertainment venues with 3,000-3,500 FSW in Liuzhou in 2010. The population of Liuzhou is estimated at 930,000 local people plus an estimated 300,000 migrants. Since 2005, the WHC's outreach 
activities have consisted of training peer educators, disseminating information and education communication (IEC) materials, and conducting HIV/STI testing for FSW every 3 months.

\section{Procedures}

We classified FSW venues into three categories based on the number of FSW employed: small ( $<10$ FSW), middle-sized (10-20 FSW), and large ( $>20$ FSW). In September 2009, after consultation with the WHC, we randomly selected three large, four middle-sized, and seven small venues from the WHC's city-wide list of 492 venues with the expectation of recruiting at least $15 \mathrm{FSW}$ from each sized venue. We then approached these venues to recruit women through the Liuzhou investigators' existing working relationships with FSW and their bosses. All 14 venues that were approached agreed to participate in the study. Women who worked in these venues and self-reported exchanging sexual services for financial compensation were eligible for inclusion. All participating FSWs came from one of the 14 study venues. Within these venues, recruitment used a combination of convenience and snowball sampling (peer referral). Each interviewed FSW was given 80 RMB ( $\$ 13$ USD) as remuneration and asked to refer other FSW peers for recruitment. 55 FSW were approached; 48 participated and 7 declined to be interviewed. Reasons for declining included hesitance to be involved $(n=3)$, disinterest $(n=2)$, and lack of time $(n=2)$. Table 1 shows the venues and number of women approached and recruited. All procedures were approved by the IRB of Renmin University of China.

After obtaining informed consent, two female graduate students from the Institute of Anthropology, Renmin University of China, collected data using questionnaires and individual, in-depth interviews. In addition to their standard discipline training, both interviewers had completed a social research methods course in which they learned how to conduct systematic interviews. Before joining our research team, both interviewers had also participated in a project in which they interviewed female migrant workers to evaluate the effectiveness of a health education program. The interviews conducted for this study lasted 1.5 to 2 hours and were conducted in a private room at the participants' workplace, or another place chosen by the participant. Each interview session included a brief selfadministered close-ended demographic questionnaire including age, education, marital status, birth place, living situation, income level, free time activities, HIV/STI knowledge, sources of knowledge, condom use, and health-seeking behaviors. For those FSW who were illiterate, the interviewer read the questions to the respondent. Two trained research assistants coded and entered the quantitative survey data into a database with initial intercoder reliability $>96 \%$.

Following completion of the questionnaire, semi-structured in-depth interviews were conducted following a set of questions focused on the women's health practices and methods for maintaining health and preventing disease, and health-seeking behaviors. (Full interview guide available on request.) Detailed notes were taken during each interview and 37 interviewees also consented to audio recording. With the permission of venue bosses and female employees, the interviewers also conducted semi-structured observations in all of the venues by recording descriptions of the environment, the activities of the FSW who were with clients, and the interactions between FSW and their bosses. The interviewers wrote field notes after each interview and observation session. For each venue category (small, medium, large), recruitment continued until interviewers reached consensus that they were no longer hearing new examples of women's sexual health practices during the in-depth interviews (i.e., saturation). At this point, two to three additional interviews were conducted within each venue category to ensure saturation.

Two of the four WHC staff were also randomly selected for audio-recorded interviews. These interviews focused on how WHC staff interacted with venues and FSW, their 
impressions of the differences between venues, and the main health problems of FSW who use WHC's free HIV/STI testing services.

Recorded interviews were transcribed by the interviewers. The co-investigator from the Liuzhou CDC supervised the interviewers and monitored the quality of the interviews and transcriptions during the data gathering phase of the project.

Our analysis was broadly informed by an interpretive interactionism perspective. As described by Denzin [22], interpretive interactionism is used "to examine the relationships between personal troubles... and the public policies and public institutions that have been created to address those troubles" (p. 2). This theoretical approach facilitated understanding the interactions between FSWs' behavioral practices, sex work environments, and the WHC and CDC activities that are meant to intervene in these women's sexual health. Interpretation occurs on both sides of these interactions as health workers categorize FSWs' behaviors into levels of sexual risk, and FSW absorb health promotion materials and messages and make decisions about how to incorporate them (or not) into their lives. For example, infertility is a common concern among FSW and for some women unwanted pregnancy is proof of continued fertility [11]. Thus, while WHC staff might see a FSW's recent abortion as evidence of continued failure to use condoms, the woman herself may interpret the need for an abortion as evidence of reproductive health in spite of her sex work and repeated STIs. The interpretive analytic approach also allows for understanding another layer of interaction as FSWs' behaviors are shaped within the constraints and characteristics of various sex work environments.

Guided by this theoretical approach, the full research team contributed to data analysis in an iterative consensus process. The two principal investigators first read the transcripts and fieldnotes, highlighted quotes in the text that described health beliefs, sexual behaviors, HIV/STI risk and HIV/STI prevention, and then applied descriptive codes to these highlighted quotations. The full research team then discussed these descriptive codes and worked together to group and develop them into themes. Based on our a priori hypothesis about the importance of the sex work environment, we then looked at how these themes were similar and different across three types of sex work environments (small, medium and large venues). In this paper we present these similarities and differences across work environments within the themes that emerged as most critical for informing HIV/STI intervention design and delivery: knowledge about sexual health, condom use, and other sexual health practices. Quotations were then chosen to illustrate both the typicality and variety within each theme. Microsoft Word and Excel matrices were used to facilitate this process.

\section{Findings}

\section{Demographic Profile of FSW Respondents}

Forty-eight FSW completed the questionnaire and interview. As can be seen in Table 2, the mean age of the sample was 25.8 years old (range 16-48). Most women could read and write, 16 had more than 9 years of education while two had no formal education. More than half $(60.4 \%)$ had been selling sex for more than 1 year.

\section{Description of Venues by Size}

The small venues ( $<10 \mathrm{FSW}$ ) typically were disguised as massage parlors or hair-salons. The 18 respondents in the small venues were between 32 and 45 years old, often divorced (10 of the 18 women), and with little formal education. Usually, the women provided their clients nothing more than sex. The sex workers in this category typically worked from 2 
p.m. to midnight and earned the least of the interviewed FSW (1,000-2,000 RMB, about $\$ 170-\$ 340$ USD per month).

In contrast to the larger venues, the small venues were loosely organized. Typically, one of the FSW also served as a boss ("mommie") for the other women. She collected a small portion from each of the other women's transactions or a certain amount every month. Working times were not strictly regulated, so the women in these venues could begin and end work when they wanted. Most of the FSW in the small venues worked long hours, however, to make more money. The clients who visited these venues were usually older and not as selective about the FSW's age or appearance as those who visited larger venues.

Medium-sized venues (10-20 FSW) were in larger, cleaner spaces than the venues with fewer sex workers. The FSW were relatively younger (average age 26.9 years) than those who worked in small venues; they were also trained in body massage, foot massage or other skills. They usually waited for clients in a lounge area, chatting or playing cards. When a client arrived, the receptionist led him to a small private room and asked a worker to serve him. If the client was not satisfied with the worker, he could ask for a different one. This kind of venue typically provided a sauna, various types of massage, hand-jobs or escort services that did not involve vaginal penetration; however, some venues also provided sexual intercourse. The average income at these venues was similar to the income at smaller venues; FSW who provided sexual intercourse earned more (2,500 RMB per month, about $\$ 400$ USD). Usually, FSW in these middle-sized venues worked from 1 p.m. to midnight.

The middle-sized venues were more strictly organized than the smaller venues. Typically, a manager was hired to run the brothel, arrange women for the clients, manage finances, and check on work attendance. The manager would arrange the order in which the women would be on duty. FSW who were late for work or left early could be fined or fired. Mid-size venues generally allowed 1-2 vacation days per month for each woman. The order would be changed only if the client asked for a different woman. A higher level boss would occasionally visit to check on the management of the venue.

The large venues, such as high-class nightclubs or KTV (karaoke) bars, were wellestablished and generally employed 20 FSW or more. These venues were usually spacious, well-organized, elaborately decorated and included multi-media entertainment services, food and alcohol. Large venues typically did not provide sex onsite. Instead, women worked as escorts who sang, danced, and drank alcohol with clients in small private rooms. Clients could negotiate individually with their escorts to go to a hotel or other off-site location for additional sexual services. In our sample, women in large venues were younger (average age 23.1 years) than those at the smaller venues and had relatively more education. At these venues the average income was 4,000-8,000 RMB per month ( $\$ 700-\$ 1,300$ USD). FSW in the large venues typically worked from 8 p.m. to midnight. When not at work, they said they would entertain themselves by surfing on the Internet or going shopping.

Large venues typically had an on-site boss, one or two managers, a financial officer, several receptionists and waitresses, as well as more than $20 \mathrm{FSW}$. FSW were divided into two to three groups each managed by a mommie. The mommies were responsible for introducing the women to the clients, settling disputes between clients and FSW, and recruiting FSW from other venues when they were needed. The FSW's compensation was not based on the days she went to work, but rather the number of times she saw clients each week. Each FSW was expected to serve clients two to four times each week.

Most of the FSW in these venues had regular clients who would contact the FSW they were familiar with when they planned to go to the club/KTV. FSWs in large venues had fewer 
time restrictions than those who worked at midsized venues. The relationship between the FSW and their mommie was more equal than in the smaller venues, apparently because the mommies wanted to keep their workers from going to other venues. If the FSW wanted time off she could ask her mommie, who generally granted the FSW's request unless a busy weekend was expected.

\section{Venue Size Differences in Knowledge About Sexual Health}

As is shown in Table 2, all but two of the FSW interviewed had heard of "HIV/AIDS." Most of them $(n=42)$ had learned about HIV from WHC outreach workers, TV/Magazines ( $n=$ $26)$ and peers $(n=15)$. The WHC used different strategies for HIV prevention based on differences they perceived in the venues. In the large and some of the middle-sized venues, the WHC organized trainings and disseminated information, education, and communication (IEC) materials before the FSW typically began work. In the small venues, the WHC staff recruited FSW who were outgoing and willing to communicate with others in their venues and trained them as peer educators. These women were asked to talk with their peers about HIV/STI in their everyday interactions. Peer educators who disseminated IEC materials and condoms received 50 RMB (\$7 USD) per day, for up to 3 days a month for their work. These peer educators were generally well received. Most of the small venue FSW said they learned from the peer educators about HIV transmission routes and that condoms could prevent them from getting HIV.

In contrast, the IEC materials alone were not well received. FSW from small venues said the materials were not useful because they were hard to understand and the content was considered irrelevant. As one 35-year-old FSW who worked in a small hair salon said: "They (IEC materials) are unreadable and hard for me to understand. I seldom read them. Besides, I am experienced and I knew how to keep myself safe long before I entered this (profession). I do not need the materials." Some women mentioned that they were not accustomed to reading in general.

Additionally, there was a mismatch between women's information interests and the IEC materials. For example, some women from the small venues wanted to learn more about reproductive health and STIs but were less interested in learning more about HIV because they did not see themselves as susceptible. As one hair salon worker (36 years old) explained: "No one ever got AIDS here. But fukebing and xingbing [gynecological diseases and STIs] are very common. We care more about fukebing and xingbing than HIV." A 39year-old FSW from a small venue concurred: "I have worked in this field for 5 years but have never heard about anyone who got HIV. But fukebing are very common." Similarly, a 24-year-old FSW from a middle-sized venue, said: "AIDS is far away from us, but fukebing are not." The most commonly mentioned gynecological diseases (fukebing) women described were urinary tract infections, yeast infections and syphilis.

In general, the FSW who worked in the larger venues expressed more interest in the IEC materials and learning about their sexual health. These women also typically had more formal education than their counterparts in the smaller venues. A few FSW from the large venues said they had previously learned about HIV/AIDS in school and were generally knowledgeable about health matters. Some of the women from the large and middle-sized venues said they read the IEC materials WHC provided and contacted doctors whenever they felt sick.

Others felt, as the small-sized venue workers had, that the trainings were ill timed, difficult to understand and irrelevant. As one 23-year-old who worked in a large nightclub said: "Most of us are not willing to come to work early to attend training. Training is boring for 
us. It's too professional; we cannot understand it. And the content is not directly related to our lives."

The FSW in the large and some of the middle-sized venues thought they faced different health problems than those in the smaller venues. They believed that the possibility of an STI was unlikely even if sexual intercourse was involved. Some workers said they were not worried about disease because their venues are "top grade" and the clients are mostly "wealthy and healthy."

Compared with the FSW in smaller venues, those in larger venues were more autonomous and could be more selective in choosing clients. They said they could decide to have sex or not on the basis of the client's physical appearance, which gave them a sense of control over potential danger. As one 21-year-old who worked in a large nightclub said:

This is a high-class venue. Most of the clients here are rich and neat. I think rich people are relatively safe. They care for their health much more than we do. If a client looks ugly or seems to be in bad health, I will refuse to go out (for sex) with him by giving an excuse, such as I am menstruating or I do not provide sex at all.

While waiting for potential clients and in their non-work time, the FSW in the larger venues spent much of their time on-line, chatting, watching films, and playing games. Their familiarity with the Internet led them to rely on it for most of the information they needed, including health. A 23-year-old FSW who worked in a large KTV bar said:

I use the Internet not only for fun but also for searching any information I need, including housing in Liuzhou, shopping, health, and so on. When I feel bad I will do key word searches on the Internet or log on the website for women to find out what the problem might be and then decide what to do next. If it is not a big problem, I would buy some medicine for self-treatment.

\section{Condom Use Varied by Venue-Size and Partner Type}

All the respondents believed that condoms were an effective way to prevent HIV infection and pregnancy and all women said they use condoms, although with varying levels of frequency. With clients, 42 of 48 FSW reported using condoms every time they had sex in the last month. Some $(n=3)$ reported that they did not use condoms every time because it was embarrassing to ask the client to use a condom when the clients patronized them regularly. As one 31-year-old FSW working in a small hair salon, said: "You know, when a client visits you many times and you get to know him better and better, he is more like a friend than a client for you. Gradually, it becomes embarrassing for you to ask for (using a condom)." In other cases, clients refused $(n=2)$ to use condoms or the client thought it was uncomfortable $(n=1)$. As one FSW (37, small hair salon) explained: "A client told me that he was uncomfortable with a condom and he would rather pay me more if I agreed to have sex without a condom. So I agreed."

FSW in larger venues appeared to have greater autonomy in negotiating condom use. As one 21-year-old FSW who worked in a large nightclub remarked: "If a client wants to (have sex with me), he must use a condom. Otherwise, I would not agree to."

Regardless of venue size, condom use was much less frequent with regular clients and partners. Among the 48 respondents, 16 were currently married, 12 were divorced, and 20 had never been married; 15 of the 20 currently unmarried women had boyfriends. Only 3 of the 31 who had a boyfriend/husband had told their husband/boyfriend about their sex work. This non-disclosure contributed to difficulty in using condoms with regular partners; only 2 of the $31 \mathrm{FSW}$ who had a boyfriend or husband said they used condoms with them. Condom 
use with a boyfriend/husband seemed to follow a cultural logic that was articulated in comments like these of a 32-year-old FSW from a middle-sized hair salon: "I did not use a condom with my husband for the first time, so never. If I ask for (using it), he may suspect that I work in this profession (i.e., sex industry), or that I would make love with others."

That almost all the FSW respondents, regardless of venue, thought it was somewhat "ridiculous" to use a condom with a husband or boyfriend suggests detachment from their involvement in the sex industry. For these FSW, sex without a condom also appeared to be an expression of trust in a close relationship.

\section{Sexual Health Practices and Health Care Differed by Venue Size}

The ages and income levels of the FSW, and the kind of services provided in different-sized venues lead to different strategies in seeking health care and sexual health practices.

Apparently because of their relatively lower incomes and greater responsibility for supporting their families than FSW in larger venues, the typically older women in the smaller venues put higher priority on continuing to make money by preventing infection and pregnancy than the younger women in the larger venues. A number $(n=30)$ of the FSW from small and mid-sized venues said they washed their vaginas before/after sex using products such as "Jieeryin" (which translates as "Clean genitals") and "Fuyanjie" ("Clean women's infection"). One FSW, 34, who worked in a small hair salon, described her regimen: "Every time I serve a client, I use wet paper towels to clean my vagina; and after work, I clean it with a kind of cleaning agent." Some women believed that washing their vaginas in this way could effectively "kill" semen and bacteria. Others acknowledged that washing was ineffective for disease prevention but still felt compelled to follow these practices.

Women who believed in the preventive efficacy of washing as well as those who acknowledged its possible ineffectiveness emphasized that these practices made them feel "cleaner" or "safer." As a 34-year-old woman from a small hair salon explained: "Doctors from the CDC told us that it's useless to clean (the vagina) after sex. They asked us to use condoms every time during sex. I try to use it (condoms). But at the same time, I would rather wash my vagina to make myself feel safer."

Some FSW in small venues said they would also wash their clothes, especially their underpants, in hot water and hang them to dry in the sun as a preventative measure. One 44year-old FSW from a small hair salon said: "It is very important for us to keep the vagina clean, so I bought a bowl and boiled my underwear from time to time. Only with high temperature could we kill bacteria." In contrast, women who worked in the larger venues seldom mentioned cleaning their vaginas or their clothes before or after sex.

A number of women $(n=18)$ from venues of all sizes reported using antibiotics before/after sex to protect themselves. These women bought oral antibiotics such as amoxicillin, tetracycline, and ciprofloxacin as recommended by peers or pharmacy workers. Some women were reluctant to use antibiotics too often, however, citing fear of infertility. A 17year-old FSW from a large KTV venue stated: "It does not matter if you use antibiotics after sex occasionally. But if you use them regularly, they will hurt your health and make you infertile."

Infertility was a common concern among many of the younger women in our sample (those primarily at large-sized venues). In addition to fears about the impact of STI or antibiotics on fertility, some women expressed concern about oral contraceptive use. One woman who worked in a large nightclub explained: 
I heard from others that oral contraceptives hurt one's health and may lead to infertility. I seldom take oral contraceptives because I want to have a baby with my boyfriend after we get married. My boyfriend and I use condoms when we make love, but not all the time. I am happy that I can (still get pregnant). (21-year-old who had had three abortions).

Due to China's current one-child family planning system and prevailing norms, few women may have multiple children or children out of wedlock. Nevertheless, for the young FSW, a pregnancy event often brought relief as an indication of continued fertility.

Many of the women in our sample had had one or more abortions. Among the 18 respondents from large venues, 14 had been pregnant at least once and 11 had had at least one abortion. The women frankly discussed their use of abortion, both self-induced and clinic based. As one 20-year-old from a large KTV venue explained: "I was found pregnant three weeks ago. I tried to get an abortion by drinking alcohol every day. But it has not worked. I may have to see a doctor later." For medical abortions, the women typically preferred the convenience and anonymity of small community clinics or private clinics. As one 22-year-old FSW who worked in a large nightclub said: "I went to a private clinic for my abortion. It is quick and convenient. No one asks your name or other information there. And no one cares about your marital status."

Consistent with greater attention to STI prevention, the women in the small and mid-sized venues who tended to have lower incomes and more family responsibilities also prioritized pregnancy prevention so they would not have to seek costly abortions. The older women who worked in the smaller venues were also more likely to use oral contraceptives because they were done having children.

Reactions to illness in general varied by venue size. When women in smaller venues felt sick, whether sexually- related or not, their first reaction was to buy medicine at a nearby pharmacy. If this medicine failed to alleviate their illness, and they could not go to work as usual, then they would see a doctor. In contrast, FSW in larger venues often turned to the Internet to find information. Such self-treatment was followed especially for conditions perceived as less serious. They also were more likely to seek a doctor's help immediately. As a 22-year-old FSW in a large nightclub said: "Whenever I feel in need, I will go to see a doctor. You know, money is not a problem for me. I can earn a few hundred RMB in one night. But we could not make money without good health." Thus, both economic factors and access to information played a role in shaping women's sexual health practices.

\section{Discussion}

Our research shows that FSW are not a homogeneous group. They are stratified in the kind of information and services they need and want based on the venues in which they work, their education levels, and the sexual services they provide. These data support previous studies that suggest that FSW in smaller venues, such as hair salons and massage parlors, may be at higher risk for HIV and other STI because they are less educated, make less money, have less access to medical care, and do not consistently use condoms with regular partners or clients. In contrast, the FSW in the larger venues, night clubs and karaoke bars, were younger, better educated, and had more access to medical care because they made more money, although they also did not consistently use condoms with their regular partners, as evidenced by their high frequency of abortions.

Regardless of venue size, women's HIV knowledge, risk perceptions and actual behaviors were at odds. This was most clearly demonstrated through their reliance on ineffective prevention techniques such as vaginal washing, douching, or self-medicating to prevent 
infection, and lack of condom use with their regular partners. Other studies have found similar patterns of high-risk behaviors among sex workers in China $[5,7]$.

Perhaps because of their higher levels of education, the women in the larger venues were more willing than the FSW in the smaller venues to read the HIV-prevention information provided by outreach workers. In contrast, four out of five of the less well-educated women working in the smaller venues and more than half of the women interviewed in the mediumsized venues considered the IEC materials irrelevant and the education sessions inconvenient and boring.

These data suggest that further efforts to prevent the spread of HIV and other STI in China would benefit from deeper understanding of the lives of FSW in different kinds of work settings. These interviews provide some ideas about how to best approach FSW in different kinds of venues with sexual health information and support. For example, the younger women in the larger venues spent much of their spare time surfing the Internet. A number said they used the Internet as a source for medical information before going to a doctor. Further inquiry into which online sites the women use most frequently and find most credible could lead to making sure those sites provide accurate information. The sites could also provide referrals to qualified medical providers.

The less well-educated and older FSW in the smaller venues were more likely to say they learned about HIV/AIDS from peer educators. Thus, peer education may be an effective strategy in the smaller venues. However, lack of access to health care and reliance on ineffective preventive measures such as douching and self-medication may require more nuanced and systemic solutions, such as provision of testing and services in ways that work for different kinds of FSW. Special websites and perhaps social networks of FSW could be set up online to provide consultations and health information for the women, especially those in large venues with Internet access. IEC materials should be based on the lived experiences of FSW to make the materials more relevant and readable. More information on reproductive health and gynecological diseases should be included so HIV/STI education would be seen as more relevant to FSW.

Working more closely with FSW in the different kinds of venues could help distinguish the best ways to provide information and services. Information should be relevant and presented in compelling, easy-to-understand formats, in the channels that FSW use. Testing and medical services should be convenient, affordable and appealing to women of different education and income levels. The Community Based Participatory Research (CBPR) approach that has been effective for developing HIV-prevention programs in other countries [23] could be helpful in developing new strategies among FSW in different kinds of venues in China. CBPR requires that relevant community members are involved not only in defining the problem but also in devising and implementing the solution. One example in rural China suggests that such a strategy could be successful. Wong and colleagues [24] described the successful revamping of an underutilized HIV/STI clinic for commercial sex workers in Ruili, China. Based on interviews with 89 local sex workers, the clinic's hours were changed, the staff was re-trained, and sex workers began coming to the clinic.

\section{Conclusions}

Although many of the FSW interviewed in this study said they knew the fundamentals about how HIV is spread and that they should be using condoms to prevent infection, many did not practice safer sex consistently, especially with their frequent and/or familiar partners, and were engaged in other ineffective prevention practices. Given that the FSW in this study did not find current education efforts relevant or convenient in time or place, more effective 
interventions are needed. CBPR with FSW in different venues could be a valuable strategy for learning which prevention approaches are most effective. Increasing basic knowledge is only one of the goals for HIV/STI prevention efforts in China. FSW, especially those working in small venues who make less money and are more likely to be supporting families, will also benefit from access to affordable reproductive and sexual health care.

\section{Acknowledgments}

This study was supported by the "Partnership for Social Science Research on HIV/AIDS in China" (NIH \#R24 HD056670) and the UNC Center for AIDS Research (NIH \#P30 AI50410), and the Research Funds of Renmin University of China. KE Muessig is supported by an NIH institutional training grant (5T32AI007001-35). We are grateful to Dr. Gail Henderson from the Department of Social Medicine, the University of North Carolina at Chapel Hill, for her support and advice during the study. The R24 team, Dr. Pan Suiming and Dr. Huang Yingying from Renmin University of China, made the study possible. We thank Malena Rousseau at the University of North Carolina at Chapel Hill for editorial assistance with the manuscript. We also are deeply grateful to the study's participants in Liuzhou.

\section{References}

1. Hong Y, Li X. Behavioral studies of female sex workers in China: a literature review and recommendation for future research. AIDS Behav. 2008; 12(4):623-36. [PubMed: 17694431]

2. Wang Q, Yang P, Gong X, et al. Syphilis prevalence and high risk behaviors among female sex workers in different settings. Chin J AIDS STD. 2009; 4:72-5.

3. Wang H, Reilly KH, Brown K, et al. HIV incidence and associated risk factors among female sex workers in a high HIV-prevalence area of China. Sex Transm Dis. 2012; 39(11):835-41. [PubMed: 23064531]

4. Chen XS, Liang GJ, Wang QQ, et al. HIV prevalence varies between female sex workers from different types of venues in Southern China. Sex Transm Dis. 2012; 39(11):868-70. [PubMed: 23064536]

5. Xia G, Yang X. Risky sexual behavior among female entertainment workers in China: implications for HIV/STD prevention intervention. AIDS Educ Prev. 2005; 17(2):143-56. [PubMed: 15899752]

6. Huang, Y. Female sex workers in China: Their occupational concerns. In: Jun, J.; Worth, H., editors. HIV in China: Understanding the social aspects of the epidemic. 43-66. Sydney: UNSW Press; 2010.

7. Hesketh T, Zhang J, Qiang DJ. HIV knowledge and risk behaviour of female sex workers in Yunnan Province, China: potential as bridging groups to the general population. AIDS Care. 2005; 17(8): 958-66. [PubMed: 16176892]

8. Chen H, Cheng F, Luan R. A study on awareness of STD/AIDS risk behaviors and health careseeking behaviors among female FSWs. Chin J AIDS STD. 2003; 6:355-7.

9. Li N, Li Z, Fan J, Huang Z, Zeng Z, Xu S. Analysis on condom use and its determinants among female sex workers in cheap entertainment places in Hefei. Chin J Dis Control Prev. 2009; 13(4): 417-9.

10. Luo Y, Lai W, Deng B. Survey of AIDS-related behavior of sex workers in Sichuan Province. Modern Prev Med. 2010; 37(2):210-2.

11. Pan, S. Survive and Experience: Tracking an Underground "Red Light Area" (Shengcun yu tiyan: dui yige dixia "hongdengqu" de zhuizong diaocha). Beijing: Chinese Social Science Publishing House; 2000.

12. Wang J, Wang T, Cen Y. Influencing factors of condom use and HIV infection in female sex workers in Zhongshan City. South China J Prev Med. 2010; 36(4):26-8.

13. Huang Y, Henderson GE, Pan S, Cohen MS. HIV/AIDS risk among brothel-based female sex workers in China: assessing the terms, content, and knowledge of sex work. Sex Transm Dis. 2004; 31(11):695-700. [PubMed: 15502679]

14. Weir S, Gandhi A, Li J, Chen XS. Entertainment and service venues in Liuzhou, China: Environments for sexual risk and syphilis prevention. In preparation. 
15. Sun X, Wang N, Li D, et al. The development of HIV/AIDS surveillance in China. AIDS. 2007; 21(Suppl 8):S33-8. [PubMed: 18172389]

16. Pan, S. A True Record of China's Red Light Distrcts (Zhongguo hongdengqu jishi). Beijing: Qunyan chubanshe; 1999.

17. Poon AN, Li Z, Wang N, Hong Y. Review of HIV and other sexually transmitted infections among female sex workers in China. AIDS Care. 2011; 23(S1):5-25. [PubMed: 21660747]

18. Rou K, Wu Z, Sullivan SG, et al. A five-city trial of a behavioural intervention to reduce sexually transmitted disease/HIV risk among sex workers in China. AIDS. 2007; 21:S95-101. [PubMed: 18172399]

19. Wong WCW. Acceptability study of sex workers attending the HIV/AIDS clinic in Ruili China. Asia Pac J Public Health. 2003; 15(1):57-61. [PubMed: 14620499]

20. Lu F, Jia Y, Sun X, et al. Prevalence of HIV infection and predictors for syphilis infection among female sex workers in southern China. Southeast Asian J Trop Med Public Health. 2009; 40(2): 263-72. [PubMed: 19323011]

21. AIDS Working Committee of Liuzhou. Working Report of HIV/AIDS Prevention and Control of Liuzhou: Working Committee of Liuzhou HIV/AIDS Prevention. Jan. 2008

22. Denzin, NK. Interpretive Interactionism. 2nd. Thousand Oaks: SAGE Publications, Inc.; 2001.

23. Rhodes SD, Hergenrather KC, Montaño J, et al. Using community-based participatory research to develop an intervention to reduce HIV and STD infections among Latino men. AIDS Educ Prev. 2006; 18(5):375-89. [PubMed: 17067250]

24. Wong WCW, Yilin W. A qualitative study on HIV risk behaviors and medical needs of sex workers in a China/Myanmar border town. AIDS Patient Care STDS. 2003; 17(8):417-22. [PubMed: 13678543] 
Table 2

Demographic characteristics, condom use, sexual health knowledge and practices of FSW in Liuzhou, China (from questionnaire) $(n=48)$

\begin{tabular}{|c|c|c|}
\hline Variable & $n$ & $\%$ \\
\hline \multicolumn{3}{|l|}{ Age (years) } \\
\hline $16-21$ & 14 & 29.2 \\
\hline $22-27$ & 16 & 33.3 \\
\hline$>27$ & 18 & 37.5 \\
\hline \multicolumn{3}{|l|}{ Education } \\
\hline No education & 2 & 4.2 \\
\hline Can read and write & 30 & 62.5 \\
\hline High school or more & 16 & 33.3 \\
\hline \multicolumn{3}{|l|}{ Origin } \\
\hline Liuzhou & 15 & 31.3 \\
\hline Non-Liuzhou & 33 & 68.7 \\
\hline \multicolumn{3}{|l|}{ Marital status } \\
\hline Never married & 20 & 41.7 \\
\hline Married & 16 & 33.3 \\
\hline Divorced & 12 & 25.0 \\
\hline \multicolumn{3}{|l|}{ Have children } \\
\hline Yes & 14 & 29.2 \\
\hline No & 34 & 70.8 \\
\hline \multicolumn{3}{|l|}{ Heard of HIV/AIDS } \\
\hline Yes & 46 & 95.8 \\
\hline No & 2 & 4.2 \\
\hline \multicolumn{3}{|l|}{ Source of knowledge ${ }^{a}$} \\
\hline Outreach of CDC & 42 & 87.5 \\
\hline TV/magazine & 26 & 54.2 \\
\hline Peers & 15 & 31.3 \\
\hline Clients & 5 & 10.4 \\
\hline Family member & 3 & 6.3 \\
\hline \multicolumn{3}{|l|}{ Protection practices during sex work ${ }^{a}$} \\
\hline Using condom regularly & 48 & 100.0 \\
\hline Washing/cleaning vagina after sex & 30 & 62.5 \\
\hline Antibiotics before/after sex & 18 & 37.5 \\
\hline \multicolumn{3}{|l|}{ Consistency of condom use with clients } \\
\hline Used condom every time for sex in the last month & 42 & 87.5 \\
\hline Use condoms but not every time in the last month & 6 & 12.5 \\
\hline \multicolumn{3}{|l|}{ Reasons for not using condom } \\
\hline Client refusal & 2 & 4.2 \\
\hline Familiar with clients & 3 & 6.3 \\
\hline Uncomfortable & 1 & 2.1 \\
\hline
\end{tabular}




\begin{tabular}{lrr}
\hline Variable & $\boldsymbol{n}$ & $\%$ \\
\hline Use condom with husband/boyfriend $b$ & 42 & 95.4 \\
$\quad$ Never use & 2 & 4.6 \\
$\quad$ Use & & \\
\hline$a_{\text {Total response exceeded } 100 \% \text { because of multiple responses }}$ & \\
$b_{4}$ respondents did not have boyfriend/husband &
\end{tabular}

